\title{
Journal of Neurological Disorders
}

\section{Essential Tremor: A Comprehensive Overview}

\section{Bhupendra Shah*}

Department of Internal Medicine, B.P. Koirala Institute of Health Sciences (BPKIHS), Nepal

\begin{abstract}
Essential tremor is the most prevalent form of movement disorders in the world and is 10 times more prevalent than that of Parkinson's disease. Even though it is traditionally considered as a benign disease all the patients of essential tremor have tremor induced disabilities. Essential tremor which shows kinetic, postural and resting is tremor mimicker of all tremor associated disease so often misdiagnosed and mismanaged. This article is authors' endeavor to summarize the facts in different publications on essential tremor. Electronic databases like MEDLINE/Pub Med, Google Scholar, IMSEAR (Index Medicus for South-East Asia Region) and Scopemed were extensively searched with MeSH (Medical Subject Headings) terms "essential tremor", "clinical features" "pathophysiology" and "treatment" from the earliest possible date of 1966 to Feb 2017. Articles in any languages especially those published in recent years were given preference. This review deals mostly with the clinical features and evidence-based management of essential tremor.
\end{abstract}

Keywords: Essential tremor; Clinical features; Treatment

\section{Introduction}

Essential tremor is considered as the most prevalent form of movement disorder in the world [1]. Its prevalence is $0.9 \%$ for all ages. Above age 40, 65 and 95 its prevalence increases to $4 \%, 4.6 \%$ and $21.7 \%$ respectively [2]. In the USA $2.2 \%$ of population has an essential tremor which is 10 times more than that of Parkinson's disease [1]. Even though traditionally it is considered as benign tremor all the patients of essential tremor have tremor induced disabilities and feelings social embarrassment [2].

\section{Methods}

Electronic databases like MEDLINE/PubMed, Google Scholar, IMSEAR (Index Medicus for South-East Asia Region) and Scope med have extensively searched with Mesh (Medical Subject Headings) terms like "essential tremor", "clinical features", "pathophysiology" and "treatment" from the earliest possible date of 1966 to Feb 2017. Articles in any language especially those published in recent years were given preference.

\section{Pathophysiology}

The evidence had shown the link between the cerebellum and essential tremor. In the last few years, advanced neuroimaging has tried to confirm this evidence. Indeed, the vast majority of studies have found functional and structural abnormalities in several parts of the anterior and posterior cerebellar lobules, however, to what degree of these neural changes contribute to clinical symptoms of essential tremor, is not established yet [3]. Though the exact pathophysiology of essential tremor is not known, one of the proposed theory for the pathophysiology of essential tremor is GABAergic dysfunction in the cerebellar dentate nucleus and brainstem caused by neurodegeneration in these regions that lead to tremulous activity within the cerebellothalamocortical circuit [4]. Functional imaging studies have suggested that tremors in ET are of central in origin associated with olivocerebellar and cerebellothalamo-cortical pathways, Positron emission tomography studies have indicated the role of gamma-aminobutyric-acid dysfunction in tremor generation while most of the structural imaging studies along with magnetic resonance spectroscopic imaging have pointed toward neurodegeneration [5]. Currently, advanced neuroimaging has confirmed the involvement of the cerebellum in pathophysiological processes of ET though there is high variability in results. For this reason, the translation of this knowledge of pathophysiology of essential into daily clinical practice is partially limited, although new advanced multivariate neuroimaging approaches are now proving interesting changes in different perspectives of the pathophysiology of essential tremor [3].

\section{Clinical Features}

Essential tremor shows autosomal dominant inheritance pattern though some sporadic cases are also reported. Its prevalence increases with age and shows a bimodal pattern of distribution. The most common body part involved in essential tremor is upper limbs (95\%) followed by head (34\%), lower limbs (20\%), voice (12\%) face $(5 \%)$ and trunk (5\%) which exhibit a mixed postural and kinetic tremor without other neurologic abnormalities [6]. The tremor is mostly progressive and symmetrical. Hand tremor which is greater at the wrist joint may be kinetic or postural usually precedes the head tremor. The amplitude of kinetic tremor is more than that of postural tremor. In the upper limbs amplitude of wrist, tremor is greater than that of metacarpal joint tremor and they show flexion extension type of movement. Intention tremor can occur in 50 percent of cases. On drawing the Archimedes spiral single orientation axis is identifiable. Head tremor is another feature of essential tremor more common in women which resolve in a supine position which shows either yes-yes or no-no phenomenon [7]. The most important factors associated with increased tremor severity are older age, longer disease duration and the presence of voice tremor [8]. Table 1 shows diagnostic criteria for essential tremor [9]. Essential tremor can mimic any other tremor associated disease like enhanced physiological tremor, Parkinson's disease and tremor associated with peripheral neuropathy. It should be differentiated from the Parkinson disease as there is a vast difference in the management of two diseases. The difference between two conditions is shown in Table 2 .

*Corresponding author: Bhupendra Shah, Department of Internal Medicine B.P. Koirala Institute of Health Sciences (BPKIHS), Nepal, Tel: + 9779842560220 E-mail: bhupendra.shah@bpkihs.edu

Received April 19, 2017; Accepted May 23, 2017; Published May 28, 2017

Citation: Shah B (2017) Essential Tremor: A Comprehensive Overview. J Neurol Disord 5: 343. doi:10.4172/2329-6895.1000343

Copyright: (c) 2017 Shah B. This is an open-access article distributed under the terms of the Creative Commons Attribution License, which permits unrestricted use, distribution, and reproduction in any medium, provided the original author and source are credited. 


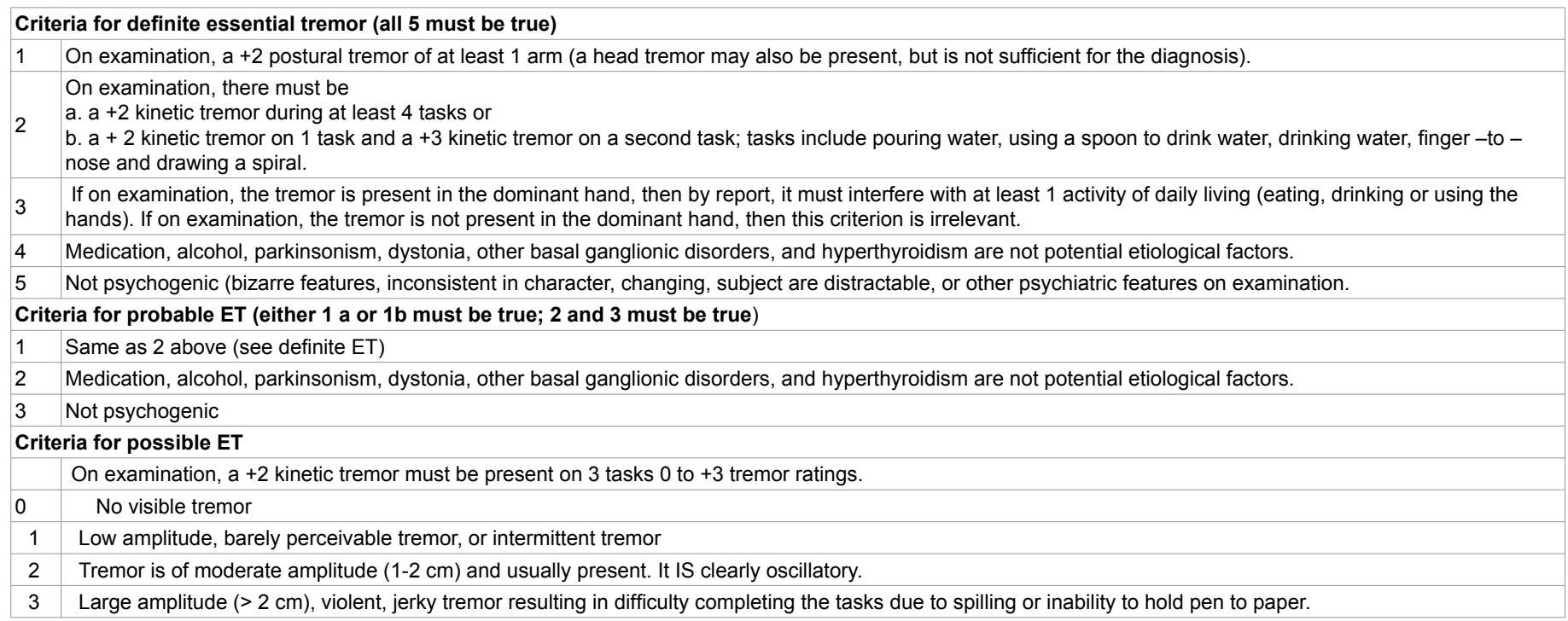

Table 1: Diagnostic criteria for essential tremor [9].

\begin{tabular}{|c|c|c|}
\hline Characteristics & Essential tremor & Parkinson disease \\
\hline Type & Kinetic, Postural tremor & Resting tremor \\
\hline Progression & Slow progression & Rapid progression \\
\hline Symmetricity & Symmetrical & Asymmetrical \\
\hline Body part involved & Hand, head, voice & Hands, legs, tongue \\
\hline Effect of alcohol & Improves & No improvement \\
\hline
\end{tabular}

Table 2: Difference between essential tremor and Parkinson disease.

\section{Laboratory evaluation}

Accelerometry of $95 \%$ of a patient of essential tremor showed frequency of $5-8 \mathrm{~Hz}$ whereas in $95 \%$ of Parkinson's disease showed the frequency of $4-8 \mathrm{~Hz}$ [10]. Archimedes spiral is another tool to differentiate the Essential tremor from Parkinson disease where single axis and multiple axes are seen in essential tremor and Parkinson disease respectively [11].

\section{Treatment}

Even though essential tremor doesn't shorten the lifespan of the individual, it makes person social disable and may have a great psychological impact. Treatment of essential tremor should be individualized based on the severity of tremor, functional impairment caused by tremor, co-morbid conditions of the patient and patient preference for treatment. The management of essential tremor includes.

- Behavioral techniques and physical therapy

- Medical therapy

- Surgical treatment [12]

\section{Behavioral techniques and physical therapy}

Essential tremor is a benign but progressive condition. There is no definitive treatment for a cure for it till now so the patient should be counseled regarding the course of the disease, impact of anxiety on tremor and expected response to treatment. Handling embarrassment and the social effects of tremor have been highlighted by ET patients as one of the major issues not being addressed in their health care [13]. Depressive symptoms are common in patients with essential tremor and have more embarrassment thus earlier treatment of depressive symptoms in ET patients might be a better strategy to lessen the burden of social embarrassment [14]. These include relaxation therapies and reducing emotional stress, using the less disabled hand to write or eat, using wrist weights and minimizing exposure to tremorogenic foods (eg; caffeine) and drugs (e.g. sympathomimetics) [12].

\section{Medical therapy}

Beta blockers: Propranolol is first line therapy for essential tremor (level A). It is only one beta blocker approved by united states FDA for the treatment of essential tremor. The mechanism of antitremor effect of the drug is blocking effects of peripheral beta- 2 receptors which are located in muscle spindles. The starting dose of propranolol is $20 \mathrm{mg} \mathrm{BD}$ which can be increased up to $320 \mathrm{mg} /$ day. The response of drug is seen in 50\% individual [15]. Long-acting propranolol is superior in efficacy than conventional propranolol in the management of essential tremor. Better compliance with once a daily dose offers an advantage over the short-acting propranolol [16]. Acute adverse reactions occurred in $8 \%$ of patients and chronic side effects seen in $17 \%$ of patients with propranolol [17]. Common side effects noticed are a headache, bradycardia, fatigability, sleep disturbances, and lightheadedness. Contraindication of beta blockers are a congestive cardiac failure, bronchial asthma, atrioventricular block and peripheral vascular disease Other beta blockers like atenolol, Sotalol and metoprolol also decrease the tremor and useful in a patient where non-selective beta blockers are contraindicated though its efficacy is lower than that of propranolol in essential tremor (level B) [18].

Primidone: Primidone is an anticonvulsant that is metabolized to phenobarbital and phenyl- ethylmaleimide. It reduces high-frequency repetitive firing of neurons and alteration of transmembrane sodium and calcium channels ion movements which is the probable mechanism of antitremor activities [15]. It can be an alternative to propranolol when beta-blockers are contraindicated in the management of essential tremor (Level A) [19]. The starting dose of primidone is $50 \mathrm{mg} / \mathrm{day}$ which can be increased up to $750 \mathrm{mg} /$ day. The response rate is $50 \%$ to $70 \%$. Common side effects noticed during primidone treatment are drowsiness, fatigue, nausea, vomiting and bone marrow suppression. It should be tapered after 6 months of use due to chances development of suicidal thought. It is contraindicated in porphyria, severe renal insufficiency, and advanced hepatic failure.

Benzodiazepines: Benzodiazepines potentiate GABAergic neurotransmission which results in hyperpolarization of the cell 
membrane and, thus, inhibition of action potential firing. This accounts for their anxiolytic, anticonvulsant, sedative, muscle relaxant, and likely also anti- tremorogenic effects. Even in a patient not responding to first-line therapy for essential tremors like propranolol and primidone, clonazepam may effective in controlling the tremor (Level C) [20]. Alprazolam another short acting benzodiazepine may reduce the tremor of essential tremor (Level B) [21]. Common side effects of benzodiazepines are drowsiness, cognitive impairment, and drug dependence.

Pregabalin: Pregabalin acts on the alpha 2-delta protein, an auxiliary subunit of voltage-gated calcium channels which reduces the synaptic release of several neurotransmitters thus the neuronal excitability [22]. Though pregabalin was proposed to have tremorlytic action, the effects of pregabalin for treating essential tremor are uncertain because the quality of the evidence is very low [23]. The starting dose is $50 \mathrm{mg}$ $\mathrm{BD}$ which can be increased up to $600 \mathrm{mg} /$ day. Common side effects of pregabalin are dizziness, vertigo, incoordination, balance disorder, ataxia, diplopia, blurred vision, amblyopia, tremor, somnolence, confusional state, and disturbance in attention, thinking abnormal, euphoria, asthenia, fatigue and peripheral edema [24].

Gabapentin: Gabapentin was proposed to have action on an auxiliary subunit of voltage-sensitive $\mathrm{Ca}^{2+}$ channels and reduce the neuronal excitation [25]. It can be used in the management of essential tremor if first line therapy fails (Level B) It was compared with propranolol for the management of essential tremor which showed comparable efficacy in reducing tremor from baseline in all tremor measures [26]. The initiating dose is $300 \mathrm{mg}$ TDS which can be increased up to $3600 \mathrm{mg}$ /day. Use of gabapentin as monotherapy is recommended only if there is a contraindication for propranolol and primidone. Common side effects of gabapentin are sedation, dizziness, and dry mouth.

Topiramate: Topiramate has multifactorial action and involves blockade of voltage-dependent sodium channels, potentiation of GABAergic transmission and inhibition of excitatory pathways through an action at AMPA receptor sites [27]. Topiramate is effective in the treatment of moderate to severe essential tremor (Level B). Functional improvements accompany tremor reduction, such as in motor tasks, writing, and speaking [28]. The most common treatment-limiting adverse events in topiramate-treated patients are paresthesia, nausea, concentration/attention difficulty, and somnolence.

\section{Surgical therapy}

Surgical therapy is recommended if the tremor is disabling and non-responsive or contraindicated to the medical treatment. Deep brain stimulation therapy and ultrasound thalamotomy are two recent surgical approaches for the management of the essential tremor.

Deep brain stimulation therapy: All medically refractory essential tremor patients should be considered for DBS. DBS of the Ventral intermediate area and the Posterior subthalamic area appears to be a safe and effective treatment for medically refractory ET [29]. Tremor and handwriting improvements in long-term follow-up are stable. The patients' perception of their outcome is good [30]. Side effects of deep brain stimulation are personality changes, mood disorders and risktaking behaviors, parenchymal haemorrhage and lead infection [31].

Ultrasound thalamotomy: Ultrasound thalamotomy is an option for management of medically refractory essential tremor. It reduces the disability and improved the quality of life. The most common side effect is alteration in sensation, gait disturbance, dysmetria, and ataxia [32].

\section{Conclusion}

Essential tremor is the most prevalent form of movement disorder in the world. The cerebellum is the proposed site for the pathophysiology of essential tremor. Propranolol and Primidone are first line therapy whereas in medically refractory patient deep brain stimulation therapy and ultrasound thalamotomy is showing promising therapeutic role.

\section{Acknowledgements}

The author acknowledges Prof. Dr. Sanjib Sharma, Dr. Lekhjung Thapa, Dr. Roshan Chhetri, Dr. Bijay Bartaula, Dr. Manish Subedi and Ms. Piyush Dahal for constantly motivating and guiding me to write this review article.

\section{References}

1. Louis ED, Ottman R (2014) How many people in the USA have essentia tremor? Deriving a population estimate based on epidemiological data. Tremor Other Hyperkinet Mov (NY) 4: 259.

2. Bain PG, Findley LJ, Thompson PD, Gresty MA, Rothwell JC, et al. (1994) Hereditary essential tremor. Brain 117: 805-824.

3. Cerasa A, Quattrone A (2016) Linking essential tremor to the cerebellumneuroimaging evidence. Cerebellum. 15: 263-275.

4. Helmich RC, Toni I, Deuschl G, Bloem BR (2013) The pathophysiology of essential tremor and Parkinson's tremor. Curr Neurol Neurosci Rep 13: 378.

5. Bhalsing KS, Saini J, Pal PK (2013) Understanding the pathophysiology of essential tremor through advanced neuroimaging: A review. J Neurol Sci 335: 9-13.

6. Elble RJ (2000) Diagnostic criteria for essential tremor and differential diagnosis. Neurology 54: 2.

7. Louis ED (2014) Twelve clinical pearls to help distinguish essential tremor from other tremors. Expert Rev Neurother 14: 1057-1065.

8. Putzke JD, Whaley NR, Baba Y, Wszolek ZK, Uitti RJ (2006) Essential tremor predictors of disease progression in a clinical cohort. J Neurol Neurosurg psychiatry 77: 1235-1237.

9. Louis ED, Ford B, Lee H, Andrews H, Cameron G (1998) Diagnostic criteria for essential tremor. Arch Neurol 55: 823.

10. Burne JA, Hayes MW, Fung VS, Yiannikas CBD (2002) The contribution of tremor studies to diagnosis of parkinsonian and essential tremor: A statistical evaluation. J Clin Neurosci 9: 237-242.

11. Badalà F, Nouri-mahdavi K, Raoof M (2008) The spiral axis as a clinical too to distinguish essential tremor from dystonia cases. Park Relat Disord 144 724-732.

12. Pramod KP (2011) Treatment for essential tremor. Ann Indian Acad Neuro 14: 25-28.

13. Louis ED (2015) Defining the treatment gap: What essential tremor patients want that they are not getting. Tremor Other Hyperkinet Mov 5: 1-12.

14. Elan D Louis, Stephanie C, Edward D. Huey (2016) Depressive symptoms can amplify embarrassment in essential tremor. J Clin Mov Disord 3: 11.

15. Hedera $P$, Frantisek $C$, Thomas $L$ Davis $P$ (2013) Pharmacotherapy of essential tremor. J Cent Nerv Dis 43-55.

16. Cleeves L, Findley LJ (1988) Propranolol and propranolol-LA in essential tremor: A double blind comparative study. J Neurol Neurosurg Psychiatry 51: 379-384.

17. William C. Koller, Bridget RN (1989) Acute and chronic effects of propranolo and primidone in essential tremor. Neurology 39: 1587-1587.

18. Leigh PN, Jefferson D, Twomey A, Marsden CD (1983) Beta-adrenoreceptor mechanisms in essential tremor; a double-blind placebo controlled trial of metoprolol, sotalol and atenolol. J Neurol Neurosurg Psychiatry 46: 710-715.

19. Dietrichson P, Espen E (1987) Primidone and propranolol in essential tremor: a study based on quantitative tremor recording and plasma anticonvulsant levels. Acta Neurol Scand 75: 332-340.

20. Biary N, Koller W (1987) Kinetic predominant essential tremor: successfu treatment with clonazepam. Neurol 7: 471-474.

21. Bruno (2015) Alprazolam for essential tremor. Cochrane database Syst Rev 6: 12 
22. Taylor CP, Angelotti T, Fauman E (2007) Pharmacology and mechanism of action of pregabalin: the calcium channel alpha2-delta (alpha2-delta) subunit as a target for antiepileptic drug discovery. Epilepsy Res 73: 137-150.

23. Bruno E, Nicoletti A, Quattrocchi G, Filippini G, Colosimo C, et al. (2016) Pregabalin for essential tremor. Cochrane Database Syst Rev 10: CD009682.

24. Zaccara G, Gangemi P, Perucca P, Specchio L (2011) The adverse event profile of pregabalin: a systematic review and meta-analysis of randomized controlled trials. Epilepsia 52: 826-836.

25. Taylor CP (1997) Mechanisms of action of gabapentin. Rev Neurol (Paris) 153 Suppl 1: S39-S45.

26. Gironell A, Kulisevsky J, Barbanoj M, López-Villegas D, Hernández G, et al. (1999) A randomized placebo-controlled comparative trial of gabapentin and propranolol in essential tremor. Arch Neurol 56: 475-480.
27. Perucca E (1997) A pharmacological and clinical review on topiramate, a new antiepileptic drug. Pharmacol Res 35: 241-56.

28. Ondo WG, Jankovic J, Connor GS, Pahwa R, Elble R, et al. (2006) Topiramate in essential tremor: A double-blind, placebo-controlled trial. Neurology 66: 672-677.

29. Chopra A, Klassen BT, Sted M (2013) Current clinical application of deep-brain stimulation for essential tremor. Neuropsychiatr Dis Treat 9: 1859-1865.

30. Zhang K, Bhatia S, Oh MY, Cohen D, Angle C (2010) Long-term results of thalamic deep brain stimulation for essential tremor. J Neurosurg. 112 1271-1276.

31. Cyron D (2016) Mental side effects of deep brain stimulation (DBS) for movement disorders: The futility of denial. Front Integr Neurosci 10: 17.

32. Elias WJ (2016) A randomized trial of focused ultrasound thalamotomy for essential tremor. N Engl J Med 375: 730-739. 